\title{
EFICÁCIA E SELETIVIDADE DO HERBICIDA FLUMIOXAZIN APLICADO EM PRÉ-EMERGÊNCIA NA CULTURA TRANSPLANTADA DA CEBOLA
}

\author{
DURIGAN, J. C. ${ }^{1}$; SILVA, M. R. M. ${ }^{2}$; AZANIA, A.A.P.M. ${ }^{3}$
}

\footnotetext{
${ }^{1}$ Eng. Agr. Prof. Adjunto do Departamento de Defesa Fitossanitária da FCAV-UNESP, 14870-000 Jaboticabal-SP; ${ }^{2}$ Doutoranda em Produção Vegetal da FCAV/UNESP, bolsista FAPEMA, rmalheir@fcav.unesp.br; ${ }^{3}$ Doutoranda em Produção Vegetal da FCAV/UNESP, bolsista CAPES, padua@fcav.unesp.br.
}

Resumo: O trabalho foi realizado com o objetivo de avaliar o desempenho do herbicida flumioxazin comparado aos padrões comerciais aplicados em pré-emergência na cultura transplantada da cebola. O experimento foi instalado em Monte Alto, SP, segundo o delineamento experimental de blocos ao acaso com quatro repetições. Os herbicidas utilizados foram: flumioxazin $\left(0,080 ; 0,120 ; 0,180 ; 0,240 \mathrm{~kg} \mathrm{ha}^{-1}\right)$, oxadiazon + linuron $\left(0,500+0,900 \mathrm{~kg} \mathrm{ha}^{-1}\right)$, linuron $\left(1,350 \mathrm{~kg} \mathrm{ha}^{-1}\right)$ e oxadiazon $\left(0,75 \mathrm{~kg} \mathrm{ha}^{-1}\right)$. Também foram mantidas duas testemunhas, uma com e outra sem controle das plantas daninhas durante todo o ciclo da cultura. As parcelas constaram de quatro linhas espaçadas de $0,36 \mathrm{~m}$ e três metros de comprimento cada, totalizando área de $4,32 \mathrm{~m}^{2}$. Foram consideradas úteis as três linhas centrais. A distância entre plantas na linha foi de 0,36 $\mathrm{m}$. Todos os herbicidas foram considerados excelentes para o controle de mentruz (Lepidium virginicum.), destacando-se flumioxazin (100\%) em todas as dosagens, até os 80 dias após a aplicação (DAA), com grande seletividade para a cultura. As plantas recuperaram-se bem dos sintomas iniciais do oxadiazon (a $\left.0,750 \mathrm{~kg} \mathrm{ha}^{-1}\right)$. A mistura de oxadiazon + linuron $\left(0,500+0,900 \mathrm{~kg} \mathrm{ha}^{-1}\right)$, assim como, o linuron isolado a $1,350 \mathrm{~kg}$ $\mathrm{ha}^{-1}$, promoveram fitointoxicação variando de moderada a média.

Palavras-chave: Fitotoxicidade, Allium cepa L., planta daninha, herbicida.

\section{EFFECTIVENESS AND SELECTIVITY OF FLUMIOXAZIN HERBICIDE IN PRÉ-EMERGENCY ON ONION CULTURE TRANSPLANTED}

ABSTRACT: The work was carried out to evaluate the herbicide flumioxazin performance compared to commercial patterns applied in pre-emergency in the transplanted onion culture. Field trials were carried out in Monte Alto, SP, arranged in a randomized complete block design with four repetitions. The used herbicides were: flumioxazin $\left(0,080 ; 0,120 ; 0,180 ; 0,240 \mathrm{~kg} \mathrm{ha}^{-1}\right)$, oxadiazon + linuron $(0,500+0,900 \mathrm{~kg}$ $\left.\mathrm{ha}^{-1}\right)$, linuron $\left(1,350 \mathrm{~kg} \mathrm{ha}^{-1}\right)$ and oxadiazon $\left(0,75 \mathrm{~kg} \mathrm{ha}^{-1}\right)$. Two controls also were maintained, one with and other without weed control during all the culture cycle. The portions consisted of four spaced lines of $0,36 \mathrm{~m}$ and three meters in length each, totalling 4,32 $\mathrm{m}^{2}$ area. Were considering useful the three central lines. The distance among plants in the line was $0,36 \mathrm{~m}$. All the herbicides were considered excellent for the Lepidium virginicum control, standing out flumioxazin $(100 \%)$ in all the dosages, until the 80 days after application (DAA), with great selectivity for the culture. The plants recovered well of the initial symptoms of the oxadiazon $\left(0,750 \mathrm{~kg} \mathrm{ha}^{-1}\right)$. The mixture oxadiazon + linuron $\left(0,500+0,900 \mathrm{~kg} \mathrm{ha}^{-1}\right)$ as well as isolated linuron $(1,350$ $\mathrm{kg} \mathrm{ha}^{-1}$ ), promoted fitotoxicity varying moderate to medium.

Keys words: fitotoxicity, Allium cepa L., weed, herbicide. 


\section{INTRODUÇÃO}

A cebola (Allium cepa L.) é uma espécie originária das regiões asiáticas, sendo de grande importância como condimento na culinária brasileira. É considerada relevante na exploração olerícola do Brasil, visto a produção de 1.183.886 ton em 2003 e a ocupação de 64.784 ha de área cultivada (Agrianual, 2004).

Existem vários fatores que ocasionam perdas na produção da cultura da cebola, dentre eles destacam-se os efeitos negativos da interferência das plantas daninhas. Conforme Ferreira et al. (2002), a cebola é uma das hortaliças mais sensíveis à interferência das plantas daninhas, podendo ocorrer reduções de $30 \%, 68 \%$ e $94 \%$ na produção de bulbos, quando o período da interferência após a emergência for de quatro, cinco e seis semanas, respectivamente. Essa interferência das plantas daninhas na cultura da cebola decorre, segundo Gelmini (1996), do porte baixo e desenvolvimento inicial relativamente lento da cultura. Em função da sua arquitetura, com folhas eretas e cilíndricas, essa cultura apresenta baixa cobertura da superfície do solo, permitindo a germinação das plantas invasoras em qualquer fase de seu desenvolvimento. Além de reduções na produção de bulbos da cultura da cebola, as plantas daninhas podem interferir na qualidade dos mesmos. Experimentos conduzidos por Soares et al. (2003) mostraram que a convivência com as plantas daninhas durante os primeiros 98 dias reduziu a produtividade da cebola em $95 \%$ e o peso médio de bulbos em $91 \%$. Portanto, o controle das plantas daninhas nesta cultura é de extrema importância para a garantia da produtividade.

O controle das plantas daninhas na cultura da cebola é, geralmente, efetuado através do controle químico, considerando-se que é uma cultura de ciclo relativamente longo com pequeno espaçamento entre plantas, o que dificulta a capina manual ou mecanizada. O controle químico proporciona melhores resultados na região da linha da cultura, não danifica o sistema radicular e economiza mão de obra, entre outras vantagens. Porém, segundo Gelmini (1996), diversas causas têm impedido a obtenção de resultados mais significativos no controle de plantas daninhas com herbicidas nessa cultura. Entre outras razões, a não utilização da dose preconizada, o emprego em épocas inoportunas e a escolha do produto incorreto em função da variedade e da infestação local, aparecem como as mais importantes. Portanto, o trabalho teve por objetivo avaliar a eficácia e a seletividade do herbicida flumioxazin, aplicado de forma isolada, comparado aos padrões comerciais, em pré-emergência das plantas daninhas.

\section{MATERIAL E MÉTODOS}

O experimento foi conduzido em propriedade particular localizada no município de Monte Alto - SP, situado a $21^{\circ} 15^{\prime}$ de latitude sul e $48^{\circ} 29^{\prime}$ de longitude oeste. O período de condução do experimento foi de junho a outubro de 1999. Antes da instalação do experimento foram retiradas amostras do solo para análises químicas e físicas cujos resultados encontram-se na Tabela 1.

O delineamento experimental utilizado foi o de blocos ao acaso, com quatro repetições. As parcelas foram constituídas por quatro linhas espaçadas de $0,36 \mathrm{~m}$ e com três metros de comprimento, perfazendo área de 4,32 $\mathrm{m}^{2}$. Considerou-se como área útil para colheita, as duas linhas centrais e como bordaduras, as duas externas. A distância entre plantas na linha era de $0,06 \mathrm{~m}$. Os herbicidas com suas respectivas doses, em ingrediente ativo e formulação comercial estão descritos na Tabela 2. 
Tabela 1. Caracterização física e química do solo do Sítio São Luís, Monte Alto-SP, realizada, no laboratório de análise de solo e planta da FCAV-UNESP. Jaboticabal-SP, 1999

\begin{tabular}{|c|c|c|c|c|c|c|c|c|c|}
\hline \multirow{2}{*}{\multicolumn{2}{|c|}{ Argila }} & & \multicolumn{2}{|c|}{ Limo } & \multicolumn{2}{|c|}{ Areia } & \multicolumn{3}{|c|}{ Classe Textural } \\
\hline & & & & & \multirow{2}{*}{$\begin{array}{l}\text { Fina } \\
460 \\
\end{array}$} & Grossa & & & \\
\hline \multirow{2}{*}{\multicolumn{2}{|c|}{170}} & & 60 & & & 310 & \multicolumn{3}{|c|}{ Média } \\
\hline & & & \multicolumn{2}{|c|}{ Características } & \multicolumn{2}{|c|}{ Químicas } & & & \\
\hline \multirow{2}{*}{$\begin{array}{l}\mathrm{pH} \mathrm{em} \\
\mathrm{CaCl}_{2}\end{array}$} & \multirow{2}{*}{$\begin{array}{l}\text { M.O. } \\
\mathrm{g} \mathrm{dm}^{-3}\end{array}$} & \multirow{2}{*}{$\begin{array}{l}\text { P resina } \\
\mathrm{mg} \mathrm{dm}^{-3}\end{array}$} & $\mathrm{~K}^{+}$ & $\mathrm{Ca}^{+2}$ & $\mathrm{Mg}^{+2}$ & $\mathrm{H}^{+}+\mathrm{Al}^{+3}$ & SB & $\mathrm{T}$ & \multirow[t]{2}{*}{$\mathrm{V}(\%)$} \\
\hline & & & \multicolumn{4}{|c|}{$\mathrm{Mmol}_{\mathrm{c}} \mathrm{dm}^{-3}$} & & & \\
\hline 4,5 & 11 & 53 & 6,1 & 13 & 4 & 34 & 23,1 & 57,1 & 40 \\
\hline
\end{tabular}

Tabela 2. Herbicidas testados e respectivas doses de ingrediente ativo e produto comercial, além das testemunhas que constituíram os tratamentos. Monte Alto - SP, 1999

\section{Herbicidas e testemunhas}

$$
\text { i.a. }\left(\mathrm{kg} \mathrm{ha}^{-1}\right) \quad \text { p.c. }\left(\mathrm{kg} \mathrm{ou} \mathrm{L} \mathrm{ha}{ }^{-1}\right)
$$

$\begin{array}{lcc}\text { 1. flumioxazin }^{(1)} & 0,080 & 0,160 \\ \text { 2. flumioxazin } & 0,120 & 0,240 \\ \text { 3. flumioxazin } & 0,180 & 0,360 \\ \text { 4. flumioxazin }^{(2)}+\text { linuron }^{(3)} & 0,240 & 0,480 \\ \text { 5. } \text { oxadiazon }^{(\text {linuron }} & 0,500+0,900 & 2,000+2,000 \\ \text { 6. oxadiazon } & 1,350 & 3,000 \\ \text { 7. } & 0,750 & 3,000 \\ \text { 8. Testemunha capinada } & - & - \\ \text { 9. Testemunha infestada } & - & -\end{array}$

${ }^{(1)}$ Flumyzin 500; ${ }^{(2)}$ Ronstar $250 \mathrm{BR} ;{ }^{(3)}$ Afalon SC.

O transplante da cultura da cebola foi realizado em 19/06/1999 e o cultivar utilizado foi o Mercedes. Foram feitas irrigações por aspersão e os tratamentos fitossanitários normais e necessários durante o ciclo.

Os herbicidas foram aplicados em pós-emergência da cultura, no estádio de 5 a 6 folhas, e pré-emergência das plantas daninhas, no dia 28/06/1999. As aplicações foram feitas pela manhã (9:00 horas), com um pulverizador costal manual, à pressão constante (mantida pelo $\mathrm{CO}_{2}$ comprimido) de $30 \mathrm{lbf} \mathrm{pol}^{-2}$, munido de barra com três bicos de jato plano tipo "leque" XR 11002 e consumo de calda equivalente a $200 \mathrm{~L} \mathrm{ha}^{-1}$. Durante a aplicação, a cultura estava ereta e com boa coloração, o solo possuía boa umidade, os ventos estavam intermitentes, a umidade relativa do ar era de $54 \%$, as temperaturas ambiente e do solo (a $5 \mathrm{~cm}$ de profundidade) eram de a $25^{\circ} \mathrm{C}$ e $20^{\circ} \mathrm{C}$, respectivamente.

As avaliações dos sintomas de fitointoxicação foram realizadas aos 7, 15, 30 e 49 dias após a aplicação (DAA) e de controle das plantas daninhas aos 30, 49 e 80 DAA. As contagens das plantas de cebola nas duas linhas centrais foram feitas aos 49 e 98 DAA, sendo que na última contagem avaliou-se também a produtividade.

As notas de fitotoxicidade foram baseadas em critérios recomendados pelo "European Weed Research Council" (EWRC, 1964). 
Para as avaliações visuais da comunidade infestante atribuíram-se porcentagens de controle proporcionadas pelos herbicidas, em relação à testemunha infestada. Os dados obtidos para as contagens das plantas cultivadas e produtividades dos bulbos foram submetidos à análise de variância, utilizando-se o teste F. Para a comparação de médias utilizou-se o teste de Tukey.

\section{RESULTADOS E DISCUSSÃO}

A principal planta daninha de ocorrência na área experimental foi o mentruz (Lepidium virginicum), encontrada em percentuais superiores a 73\% na testemunha infestada em todas as avaliações realizadas. Segundo Kissmann \& Groth (1999) essa espécie é infestante em hortas e jardins, eventualmente em culturas, durante os meses mais frios do ano. A planta prefere solos francos, ricos em matéria orgânica e com boa umidade, sendo resistente ao frio. Deuber (1997) destaca o mentruz como uma das espécies que se desenvolve muito bem em áreas cultivadas com hortaliças, por serem enriquecidas com materiais orgânicos e fertilizantes, além de disporem de abundante suprimento de água.

O controle desta planta daninha foi excelente em todas as avaliações e para todos os herbicidas, destacando-se a performance do flumioxazin isolado em todas as dosagens testadas $\left(0,080\right.$ até $\left.0,240 \mathrm{~kg} \mathrm{ha}^{-1}\right)$, que manteve índices de $100 \%$ de controle até 80 DAA (Tabela 3). Resultados semelhantes foram obtidos nesta cultura por Rozanski et al. (2002) com o mesmo herbicida nas dosagens de 25 g.ha ${ }^{-1}$ até 75 g.ha ${ }^{-1}$.

Tabela 3. Médias das porcentagens de controle atribuídas nas avaliações visuais de mentruz (Lepidium virginicum) na cultura da cebola, em diferentes épocas após a aplicação de herbicidas (DAA). Monte Alto - SP, 1999

Época de avaliação do controle

\begin{tabular}{lcccc}
\multicolumn{1}{r}{ Herbicidas e testemunhas } & Dosagens & \multicolumn{3}{c}{ (DAA) } \\
\cline { 5 - 5 } & $\left(\mathrm{kg} \mathrm{ha}^{-1}\right)$ & 30 & 49 & 80 \\
\hline 1. flumioxazin & 0,080 & 100,0 & 100,0 & 100,0 \\
2. flumioxazin & 0,120 & 100,0 & 100,0 & 100,0 \\
3. flumioxazin & 0,180 & 100,0 & 100,0 & 100,0 \\
4. flumioxazin & 0,240 & 100,0 & 100,0 & 100,0 \\
5. oxadiazon + linuron & $0,500+0,900$ & 100,0 & 97,5 & 95,0 \\
6. linuron & 1,350 & 100,0 & 100,0 & 97,5 \\
7. oxadiazon & 0,750 & 100,0 & 95,0 & 93,7 \\
8. Testemunha capinada & - & 100,0 & 100,0 & 100,0 \\
9. Testemunha infestada & - & $(82,5)^{*}$ & $(75,0)$ & $(73,7)$ \\
\end{tabular}

* porcentagem de infestação na testemunha.

Com relação à fitotoxicidade, cujos dados são apresentados na Tabela 4, o flumioxazin foi responsável por necroses leves nos ponteiros, com as doses de 0,080 até $0,180 \mathrm{~kg} \mathrm{ha}^{-1}$. Os sintomas foram mais acentuados, com algumas necroses e reduções de altura, na dose de $0,240 \mathrm{~kg} \mathrm{ha}^{-1}$. No entanto, em todos os tratamentos com flumioxazin 
foi observada boa recuperação aos 49 DAA. Os efeitos foram mais acentuados apenas na dosagem de $0,240 \mathrm{~kg} \mathrm{ha}^{-1}$, cujas plantas mantinham um porte levemente menor que a testemunha capinada. Experimentos conduzidos por Rozanski et al. (2002) mostraram que o herbicida flumioxazin aplicado após o transplante, em pós-emergência das plantas daninhas, mostrou boa seletividade à cultura da cebola cv. Superex.

Tabela 4. Médias das notas atribuídas nas avaliações visuais dos sintomas de intoxicação nas plantas de cebola, em diferentes épocas após a aplicação dos herbicidas (DAA). Monte Alto - SP, 1999

\begin{tabular}{lccccc}
\hline \multirow{2}{*}{$\begin{array}{l}\text { Herbicidas e testemunhas } \\
\text { Dosagens }\end{array}$} & \multicolumn{4}{c}{$\begin{array}{c}\text { Época de avaliação de } \\
\text { fitointoxicação (DAA) }\end{array}$} \\
\cline { 3 - 6 } & $\left(\mathrm{kg} \mathrm{ha}^{-1}\right)$ & 7 & 15 & 30 & 49 \\
\hline 1. flumioxazin & 0,080 & 2,2 & 2,7 & 1,2 & 1,0 \\
2. flumioxazin & 0,120 & 3,2 & 3,7 & 2,2 & 1,0 \\
3. flumioxazin & 0,180 & 3,2 & 4,1 & 3,5 & 1,7 \\
4. flumioxazin & 0,240 & 4,0 & 4,5 & 4,0 & 2,7 \\
5. oxadiazon + linuron & $0,500+0,900$ & 5,5 & 5,7 & 6,0 & 5,0 \\
6. linuron & 1,350 & 4,2 & 5,7 & 5,7 & 4,5 \\
7. oxadiazon & 0,750 & 4,0 & 2,7 & 2,0 & 1,0 \\
8. Testemunha capinada & - & 1,0 & 1,0 & 1,0 & 1,0 \\
9. Testemunha infestada & - & 1,0 & 1,0 & 1,0 & 1,0 \\
\hline
\end{tabular}

Para a mistura em tanque de oxadiazon + linuron $\left(0,500+0,900 \mathrm{~kg} \mathrm{ha}^{-1}\right)$ os sintomas foram mais drásticos, com queimaduras de folhas, principalmente dos ponteiros, chegando à eliminação de plantas devido às extensas necroses, além da redução de altura. O linuron isolado $\left(1,350 \mathrm{~kg} \mathrm{ha}^{-1}\right)$ provocou sintomas bastante parecidos com os da mistura em tanque. Leal (1984), estudando o controle químico de plantas daninhas na cultura da cebola, observou que nos tratamentos que se incluiu o herbicida linuron ocorreram reduções drásticas na altura e fortes sintomas de intoxicação nas plantas.

Para o tratamento com oxadiazon $\left(0,750 \mathrm{~kg} \mathrm{ha}^{-1}\right)$ isolado, apesar dos sintomas de queimaduras de ponteiros, além de leve redução de altura, ocorreu recuperação das plantas a partir dos 15 DAA.

A menor seletividade da mistura oxadiazon + linuron $\left(0,500+0,900 \mathrm{~kg} \mathrm{ha}^{-1}\right) \mathrm{e}$ linuron isolado $\left(1,350 \mathrm{~kg} \mathrm{ha}^{-1}\right)$ ficou evidente na Tabela 5 , com os dados do número de plantas nas duas linhas centrais, aos 49 e 98 DAA (por ocasião da colheita) e diferiram com relação à testemunha capinada. Leal (1984) também obteve produtividades menos expressivas com a produção de cebola nas misturas com linuron. Gomes (1987) citado por Ferreira (1997), ao avaliar combinações de doses de linuron aplicadas em pré e pósemergência das plantas de cebola, obteve aumentos na produção de bulbos com aplicações de até $0,300 \mathrm{~kg} \mathrm{ha}^{-1}$ em pré-emergência, combinadas com $0,380 \mathrm{~kg} \mathrm{ha}^{-1} \mathrm{em}$ pós-emergência. Segundo o autor, isso ocorreu devido à redução da interferência das plantas daninhas com as plantas de cebola. A partir delas a produção de bulbos diminuiu devido à redução de estande pelo efeito aditivo das doses. 
$\mathrm{Na}$ avaliação de produtividade estas diferenças não foram significativas estatisticamente, apesar dos menores valores obtidos, possivelmente pela compensação proporcionada pelos maiores bulbos destas plantas. Observa-se também uma tendência de queda da produtividade na maior dosagem de flumioxazin $\left(0,240 \mathrm{~kg} \mathrm{ha}^{-1}\right)$.

A pesquisa realizada permite concluir que todos os herbicidas usados são excelentes para o controle de mentruz (Lepidium virginicum), destacando-se flumioxazin com índices de $100 \%$, até 80 DAA, em todas as dosagens, desde 0,080 até $0,240 \mathrm{~kg} \mathrm{ha}^{-1}$. O herbicida flumioxazin foi seletivo para a cultura em todas as dosagens testadas e o oxadiazon a $0,750 \mathrm{~kg} \mathrm{ha}^{-1}$ provocou sintomas iniciais de fitotoxicidade nas plantas, dos quais recuperaram-se. A mistura de oxadiazon + linuron $(0,500+0,900 \mathrm{~kg}$ $\left.\mathrm{ha}^{-1}\right)$ e linuron isolado $\left(1,350 \mathrm{~kg} \mathrm{ha}^{-1}\right)$ promoveram fitotoxicidade, cuja toxicidade variou de moderada a media.

Tabela 5. Médias do número de plantas e produtividade de bulbos de cebola. Monte Alto - SP, 1999

\begin{tabular}{lcccc}
\multirow{2}{*}{$\begin{array}{c}\text { Herbicidas e } \\
\text { testemunhas }\end{array}$} & $\begin{array}{c}\text { Dosagens } \\
\left(\mathrm{kg} \mathrm{ha}^{-1}\right)\end{array}$ & \multicolumn{2}{c}{ Número de plantas/6 m* } & \multirow{2}{*}{ Produtividade } \\
\cline { 3 - 4 } & & 49 DAA & $98 \mathrm{DAA}$ & g/4,32 $\mathrm{m}^{2}$ \\
\hline 1. flumioxazin & 0,080 & $100,5 \mathrm{~A}$ & $95,2 \mathrm{AB}$ & $10.342,5$ \\
2. flumioxazin & 0,120 & $91,5 \mathrm{AB}$ & $91,5 \mathrm{ABC}$ & $11.702,5$ \\
3. flumioxazin & 0,180 & $85,7 \mathrm{AB}$ & $81,0 \mathrm{ABC}$ & $10.232,5$ \\
4. flumioxazin & 0,240 & $81,5 \mathrm{AB}$ & $77,5 \mathrm{ABC}$ & $9.187,5$ \\
5. oxadiazon + linuron & $0,500+0,900$ & $70,2 \mathrm{~B}$ & $69,0 \mathrm{C}$ & $8.435,0$ \\
6. linuron & 1,350 & $72,7 \mathrm{~B}$ & $72,5 \mathrm{BC}$ & $9.940,0$ \\
7. oxadiazon & 0,750 & $100,2 \mathrm{~A}$ & $100,2 \mathrm{~A}$ & $11.545,0$ \\
8.Testemunha capinada & - & $94,5 \mathrm{AB}$ & $93,5 \mathrm{AB}$ & $13.062,5$ \\
9.Testemunha infestada & - & $95,0 \mathrm{AB}$ & $90,5 \mathrm{ABC}$ & $11.177,5$ \\
\hline Teste F & & $6,41^{* *}$ & $4,94 * *$ & $2,02 \mathrm{NS}$ \\
CV (\%) & & 10,1 & 11,5 & 18,6 \\
DMS & & 21,29 & 23,74 & 4762,77 \\
\hline
\end{tabular}

* Médias seguidas de mesma letra na coluna não diferem entre si pelo teste de Tukey a $5 \%$ de probabilidade

\section{LITERATURA CITADA}

AGRIANUAL: Anuário da Agricultura Brasileira. São Paulo: FNP Consultoria, 2004.

DEUBER, R. Ciência das Plantas Infestantes - Manejo. São Paulo: Campinas, v.2, 1997. 285p.

EUROPEAN WEED RESEARCH COUNCIL - EWRC. Report of the $3^{\text {rd }}$ and $4^{\text {th }}$ meetings of EWRC: Committee of Methods in Weed Research. Weed Research, v.4, n.1, p.88, 1964.

FERREIRA L.R. Eficácia e seletividade de herbicidas para a cultura da cebola (Allium cepa L.) em semeadura direta. 1997. 130 f. Tese (Doutorado em Agronomia) - Universidade Estadual Paulista, Jaboticabal. 1999. 
FERREIRA, M. D. Cultura da cebola: Recomendações Técnicas. Asgrow Vegetable Seeds, Campinas, s.n., 2000. 36 p.

FERREIRA, L.R.; FERREIRA, F.A.; SILVA, C.M.M. da . Controle químico de plantas daninhas na cultura da cebola. Informe Agropecuário, Belo Horizonte, v.23, n.218, p.51-56, 2002.

GELMINI, G. A. Herbicidas: Indicações básicas para a cultura da cebola. Manual CATI, Campinas, n²9, 1996. 17 p.

KISSMANN, K.G.; GROTH, D. Plantas Infestantes e Nocivas. 2 ed. São Paulo: BASF, 1999. 978 p.

LEAL, F. R. Efeitos da profundidade, da densidade de semeadura direta e do controle químico em pós-emergência das plantas daninhas na produção de cebola (Allium cepa L.). 1984. 88 f. Dissertação (Mestrado em Agronomia) - Universidade Estadual Paulista, Jaboticabal, 1984.

ROZANSKI, A.; COSTA, E. A. D. da; MATALlO, M. B.; BURGA, C. A. Efeito do herbicida flumioxazin nas plantas daninhas e na cultura da cebola. Boletim Informativo, Ciência das Plantas Daninhas, SBCPD, v.8, n.1, 2002.

SOARES, D. J.; PITELLI, R. A.; BRAZ, L. T.; GRAVENA, R.; TOLEDO, R. E. B. Períodos de interferência das plantas daninhas na cultura de cebola (Allium cepa) transplantada. Planta Daninha, v.21, n.3, p.387-396, 2003. 